CASE REPORT. International Journal of Basic and Applied Medical Sciences. 2014 Vol. 4 (3), pp. 101-103.

3. Abhilash KPP, Arul JJ, Bala D. Fatal overdose of iron tablets in adults. Indian J Crit Care Med. 2013;17(5): 311-3..

4. Jover F, Andreu L, Robert J, Merino J. [Acute iron poisoning. A case report]. Rev Med Chil. 2001;129(6): 660-2.

5. Baranwal AK, Singhi SC. Acute iron poisoning: Management guidelines. Indian Pediatr. 2003;40:534-40

6. Daram SR and Hayashi PH. Acute liver failure due to iron overdose in an adult. Southern Medical Journal. 2005; 98: 241-44.

7. Robertson A, Tenenbein M. Hepatotoxicity in acute iron poisoning. Hum ExpToxicol. 2005;24:559-62.

\section{Quetiapine induced acute pancreatitis: a case report from Eastern Nepal}

Acute pancreatitis is an inflammatory condition of the pancreas with variable involvement ofextrapancreatic organs. Gallstones, alcohol and hypertriglyceridemia are the most commonly recognized etiologies of acute pancreatitis. Medications, autoimmune pancreatitis and ERCP (Endoscopic Retrograde Cholangiopancreatography) pancreatitis, apart from other rare causes, have also been implicated in the causation of the disease. Second-generation antipsychotics (SGAs) such as Quetiapine, olanzapine and risperidone, have well known metabolic side effects such as hyperlipidemia and hyperglycemia. ${ }^{1}$ Quetiapine is a widely used antipsychotic in the treatment of delirium. Although second-generation antipsychotics are associated with pancreatitis, Quetiapine-associated pancreatitis is extremely rare. ${ }^{2}$
We present what we believe to be the first report of Quetiapine induced acute pancreatitis seen at our tertiary care hospital in Eastern Nepal.

\section{Case Report}

A 32 year old male presented to the emergency room with progressive, moderate to severe epigastric pain for 3 days associated with nausea and post prandial bloating. The pain radiated to the back and right shoulder. He reported no history of fever, jaundice, vomiting of blood or dark stool. The patient reported no history of smoking or alcohol use and he consumed a vegan diet. He recently survived a major road traffic accident one and a half months ago, where he sustained a head concussion for which he was hospitalized and kept in the ICU for observation. During the hospital stay the patient developed delirium and was diagnosed to have depression on detailed psychiatric assessment. He was put on Quetiapine $25 \mathrm{mg}$ twice daily along with midazolam $5 \mathrm{mg}$ at bed time. He was on the atypical antipsychotic for one and half months before presenting with this episode of abdominal pain.

On examination he was in distress with abdominal pain. He had generalized tenderness, guarding and rigidity of the abdomen. He had not passed flatus for 3 days and his bowel sounds was sluggish. He was kept NPO (Nil per Oral),with a nasogastric tube, started on intravenous opioids and was given judicious hydration while closely monitoring his urine output. His investigations revealed high amylase $920 \mathrm{IU} / \mathrm{ml}$, lipase $1010 \mathrm{U} / 1$, hematocrit $42.5 \%$, total leukocyte count 17000 cells $/ \mathrm{mm} 3$ and hsCRP (high sensitivity C-reactiveprotein) $198.4 \mathrm{mg} / 1$. There was no evidence of hypertriglyceridemia, diabetic ketoacidosis or other metabolic abnormalities. His abdominal CT revealed moderate severity of the disease (Modified CTSI score- 4) with minimal left sided pleural effusion.

With the temporal relationship of use of Quetiapine for 6 weeks and the absence of typical etiological factors (eg. Alcohol, gallstones, hypertriglyceridemia) Quetiapine was implicated for the patient's acute pancreatitis. Psychiatric consultation was done and Quetiapine was stopped and replaced with escitalopram. The patient became medically stable after 3 days of hospitalisation. 
His amylase and lipase dropped down to $560 \mathrm{IU} / 1$ and $600 \mathrm{U} / 1$ respectively. His leukocytecount normalized and hematocrit came down to $30 \%$. He was discharged and asked to follow up after 2 weeks in the Gastroenterology Out Patient Department. On follow up he continued to improve with good medication compliance and remained symptom free.

\section{Discussion}

Acute pancreatitis has varied etiologies, though the commonly implicated ones are alcohol, gallstones and hypertriglyceridemia. Quetiapine, an atypical antipsychotic which our patient was using is prescribed along with haloperidol in the ICU for delirium. Though SGAs (Second generation antipsychotics) are known to cause metabolic disturbances like hyperglycemia and hypertriglyceridemia, complications from these metabolic side-effects, such as acute pancreatitis and diabetic ketoacidosis, are exceedingly rare. ${ }^{3}$ Quetiapine induced hypertriglyceridemia causing acute pancreatitis has been reported in only five published case reports. ${ }^{4}$

In this case, the use of quetiapine could not be linked to serious metabolic side effects. In the absence of other potential risk factors, we presumed that the development of pancreatitis could be attributed to Quetiapine. Hypertriglyceridaemiainduced acute pancreatitis from Quetiapine use does not appear to be dose dependant, but appears instead to be related to the duration of intake of the drug. All cases have been reported within the first 8 months of an established dose, most frequently at 3 months of treatment, with one report of occurrence within 72 h. ${ }^{1,4}$ The mechanism by which quetiapine causes hyperlipidemic disturbances is not entirely understood though some hypothesise altered triglyceride metabolism leading to oxidative stress ultimately causing acute pancreatitis.

SGAs also possess a well-documented side effect of blood dyscrasias, such as agranulocytosis. Leukopenia associated with quetiapine is most predominantly pronounced by selective absolute neutrophil depression, though the exact incidence is not known. ${ }^{5}$ Although our patient did not have any hematological adverse effect to the drug, presence of pancytopenia, specially neutropenia, could assist in the diagnosis of quetiapine induced pancreatitis.

Clinicians must be careful not to blame a drug when a patient with acute pancreatitis does not have an obvious underlying cause. Many of the published case reports suffer from a combination of inadequate criteria for the diagnosis of acute pancreatitis, failure to rule out more common causes, or a lack of a re-challenge with the medication. We excluded the classic causes of pancreatitis such as alcohol, gallstones, hyperglyceridemia and hypercalcemia in our patient. However we did not rechallenge the patient with the same drug to confirm the cause and effectdue to possible ethical issues attached to such an approach. Although a positive re-challenge with a drug is the best evidence available for cause and effect, it is not proof.

We conclude from our observations that while second-generation antipsychotics (SGAs) are generally safe, they can occasionally cause serious metabolic and haematologicalcomplications. Acute pancreatitis is rarely caused by medications and Quetiapine has been implicated in only a few published case reports. To our knowledge this is the first case report of Quetiapine induced pancreatitis noted here in a tertiary care hospital in eastern Nepal.

RABIN SHARMA BIJAY BARTAULA MANISH SUBEDI

Division of Gastroenterology,
Department of Internal Medicine,
BPKIHS, Dharan, Nepal

Correspondence: Dr Rabin Sharma Email:docrabinsharma@gmail.com

\section{References}

1. Duncan EJ, Woolson SL, Hamer RM, et al. Risk of lipid abnormality with haloperidol, olanzapine, quetiapine, and risperidone in a Veterans Affairs population. IntClinPsychopharmacol2009;4:204-13. 
2. Koller EA, Cross JT, Doraiswamy PM, Malozowski SN. Pancreatitis associated with atypical antipsychotics: from the Food and Drug Administration's MedWatch surveillance system and published reports. Pharmacotherapy. 2003;23(9):1123-30.

3. Potolidis E, Mandros C, Karakitsos D, et al. Quetiapineassociated pancreatitis in a geriatric critical care patient with delirium. Case Rep Psychiatry 2012;2012:625954.

4. Liou LS, Hung YJ, Hsieh $\mathrm{CH}$, et al. Aggravation of hypertriglyceridemia and acute pancreatitis in a bipolar patient treated with quetiapine. Yonsei Med J 2014;55:831-3.

5. Tang HC, Chung KH. Quetiapine-induced neutropenia in a bipolar patient with hepatocellular carcinoma. Int J Psychiatry Med 2014;47:255-61.

\section{Deep vein thrombosis in ulcerative colitis: a case report from Eastern Nepal}

Inflammatory bowel disease (IBD) including ulcerative colitis and Crohn's disease are chronic disorders of unclear pathogenesis. Traditionally considered a relatively uncommon disorder in developing nations like the South Asian countries, its incidencehas been increasingin these areas and awareness regarding the disease is now growing. Extra-intestinal manifestations of IBD are seen to occur in 25 per cent of cases. Vascular manifestations are rare and the most relevant among them are deep veinthrombosis (DVT) and pulmonary embolism (PE). The clinical course is associated with a higher incidence of inflammatory bowel disease, especially among hospitalized patients. ${ }^{1}$

Here we present what we believe to be the first report of a patient of ulcerative colitis with deep vein thrombosis as an extra-intestinal manifestation in a tertiary care center in Nepal.

\section{Case report}

A 65-year old gentleman who was a known case of ulcerative colitis (diagnosed in April 2014) presented to our Emergency department following a general practitioner referral, complaining of a swelling below the left knee, pain and superficial thrombophlebitis. The thrombophlebitis had been present for three days while the swelling and pain were of one day duration. The patient's intestinal disease was clinically in remission as evident by SCCAI $<2$ (Simple Clinical Colitis Activity Index). The patient had been receiving oral mesalamine 2.4 grams once daily fortwo years for ulcerative colitis. He had no known drug allergies.

On examination, he had a hot, swollen and tender swelling below the left knee which was painful to touch on the medial aspect and in the popliteal fossa. The remainder of the examination was normal.

Investigations revealed a raised ESR of $46 \mathrm{~mm} / \mathrm{hr}$, CRP $10.1 \mathrm{mg} / \mathrm{l}$ and D-dimer level of $3000 \mathrm{ng} / \mathrm{ml}$. Venous Doppler of the lower limb revealed a thrombus involving the left external iliac, femoral vein andpopliteal veins with thickened and edematous skin and subcutaneous tissues in the left thigh and leg. The patient also underwent an unprepared sigmoidoscopy for the assessment of disease activity of ulcerative colitis. There was loss of vascular pattern noted in the rectum which was suggestive of mild disease activity). A biopsy was sent to assess the histological disease activity.

The patient was managed with low molecular weight heparin, warfarin and compression stockings for the left leg. He was discharged after 5 days with mesalamine 2.4 grams daily in divided doses and warfarin maintaining the target INR between 2 and 3. He was on regular follow up withPT/INR report on an out-patient basis. He was reassessed with a Doppler ultrasound after 6 months which revealed a thrombus on the posterior wall of the left external iliac vein with no compromise of blood flow distally. He was also on clinical remission with mesalamine. The patient was asked to continue anticoagulation (warfarin) whle this case was being reported. 